\title{
RELACIÓN ENTRE LA DENSIDAD ESTOMÁTICA, LA TRANSPIRACIÓN Y LAS CONDICIONES AMBIENTALES EN FEIJOA (Acca sellowiana [O. BERG] BURRET)
}

\section{RELATIONSHIP BETWEEN STOMATAL DENSITY, TRANSPIRATION AND ENVIRONMENTAL CONDITIONS IN PINEAPPLE GUAVA (Acca sellowiana [O. BERG] BURRET)}

\author{
Jeison Naizaque ${ }^{1}$, Gustavo García ${ }^{1}$, Gerhard Fischer ${ }^{2}$, Luz Marina Melgarejo ${ }^{3}$
}

\begin{abstract}
${ }^{1}$ Ingeniero Agrónomo, Facultad de Ciencias Agrarias, Departamento de Agronomía, Universidad Nacional de Colombia, Bogotá (Colombia), e-mail: naizaque@hotmail.com, gagarciavi@gmail.com; ${ }^{2}$ Ingeniero Hortícola, Ph.D., Profesor Asociado, Departamento de Agronomía, Facultad de Ciencias Agrarias, Universidad Nacional de Colombia, Bogotá (Colombia), e-mail: gfischer@unal.edu.co; ${ }^{3}$ Bióloga, Ph.D. Profesora Titular, Departamento de Biología, Facultad de Ciencias, Universidad Nacional de Colombia, Bogotá (Colombia), e-mail: Immelgarejom@unal.edu.co
\end{abstract}

Rev. U.D.C.A Act. \& Div. Cient. 17(1): 115-121, Enero-Junio, 2014

\section{RESUMEN}

La feijoa es una planta con un alto grado de adaptación a las condiciones agroecológicas de la zona andina y, a pesar del aumento de la demanda nacional e internacional, son muchos los aspectos que faltan por conocer acerca de su fisiología. El presente trabajo tuvo como objetivo evaluar la tasa de transpiración, la densidad estomática y los factores ambientales asociados, en los estratos superior e inferior de la copa de árboles de feijoa, en un cultivo localizado en Tibaná (Boyacá, Colombia), bajo condiciones de campo. Se realizaron mediciones de la temperatura foliar, la tasa de transpiración, la irradiancia y la humedad relativa (HR) en los dos estratos, durante 7 horas, del día (8:00, 9:00, 10:00, 11:00 am, 12:00 m, 1:00 y 2:00 pm). En el estrato superior, se observó mayor tasa de transpiración y mayor número promedio de estomas, $91 / \mathrm{mm}^{2}$ vs. $78 / \mathrm{mm}^{2}$ del inferior. La transpiración foliar aumentó de manera directa con el incremento de la temperatura (siendo mayor en el estrato superior que en el inferior) y con la irradiancia, en tanto que aumentó a medida que disminuyó la humedad relativa.

Palabras clave: Estomas; irradiancia; humedad relativa; temperatura; estrato copa.

\section{SUMMARY}

Pineapple guava is a plant with a high adaptation to environmental conditions of the Andean region. Although there exists an increased national and international demand, there are many physiological aspects that still need to be known. This study aimed to evaluate the transpiration rate, the density of stomata, and the associated environmental factors in the upper and lower stratum of the tree crown of the pineapple guava crop in Tibaná municipality (Boyacá, Colombia), under open air conditions. Measurements were made of leaf temperature, transpiration rate, irradiance and relative humidity $(\mathrm{RH})$ in two strata during 7 daily hours (8:00, 9:00, 10:00, 11:00, 12:00, 13:00 and 14:00 h). The upper stratum showed a higher transpiration rate and a major average number of stomata, $91 / \mathrm{mm}^{2}$ vs. $78 / \mathrm{mm}^{2}$ in the lower stratum. Leaf transpiration increased directly with increasing temperature (more in the upper stratum than in the lower stratum) and with the irradiance; while transpiration increased as the relative humidity decreased.

Key words: Stomata; irradiance; relative humidity; temperature; crown stratum.

\section{INTRODUCCIÓN}

La feijoa (Acca sellowiana [O. Berg] Burret, familia Mirtaceae) es una planta arbustiva subtropical, originaria de Sud- 
américa, específicamente, de las zonas comprendidas entre el sur de Brasil, Uruguay, las partes altas del lado occidental de Paraguay y el nororiente de Argentina (Schumann \& Lüdders, 1992).

Agronet (2014) reportó, para el 2012, una producción de 1.335ton de feijoa, sobre un área total de $176 \mathrm{ha}$, en los departamentos de Boyacá (164ha), Cundinamarca (5ha), Norte de Santander (2ha) y Santander (5ha), con un rendimiento promedio de 7,6 t ha ${ }^{-1}$; sin embargo, Quintero (2012) estimó para Colombia un área sembrada de feijoa de 650ha. Este último autor recomienda para la siembra sitios en altitudes entre 1.800 y $2.700 \mathrm{msnm}$. Barrero (1993) considera que las temperaturas mínimas y máximas promedios ideales se encuentran entre 13 y $21^{\circ} \mathrm{C}$ (con un promedio de $16^{\circ} \mathrm{C}$ ). De acuerdo con Quintero (2012), la feijoa prefiere localidades con precipitaciones entre los 700 y $1.200 \mathrm{~mm}$ al año; no obstante, toleran precipitaciones de hasta $2.000 \mathrm{~mm}$ anuales, en condiciones de buena luminosidad y humedad relativa promedio del $70 \%$. Cultivos a plena exposición solar prosperan mejor, por lo cual, zonas con 1.500 horas o más de brillo solar/año son muy aptas (Quintero, 2012).

La feijoa es una planta adaptada a condiciones secas; posee hojas pequeñas, coriáceas, con cutícula gruesa y alta densidad de tricomas, que disminuyen el área transpiratoria de la planta (Fischer, 2003). Además, el color verde brillante en el haz y blanquecina en el envés de las hojas de feijoa (Fischer, 2003) disminuyen la pérdida de agua, comparada con hojas de sólo color verde oscuro (Kozlowski \& Pallardy, 1997).

Los estomas de feijoa, se encuentran ubicados en la epidermis de la hoja, en la parte abaxial (envés) (Fischer, 2003). Se ha reportado en otras especies, que el número de estomas por área de superficie foliar (densidad o frecuencia estomática), se registra entre 10 y 2.000 por $\mathrm{mm}^{2}$ (Öpik \& Rolfe, 2005).

La evaporación del agua en las hojas facilita el movimiento del agua y nutrientes en la planta (Sánchez-Díaz \& Aguirreolea, 2008); es el proceso, mediante el cual, el agua pasa a través del continuo agua-suelo-planta-atmósfera (López, 2000). En la planta, se evapora desde la superficie interna que rodea las células de las cámaras subestomáticas y pasa a través de los estomas, hacia la fase gaseosa del ambiente de la hoja (Salisbury \& Ross, 2000). Sánchez-Díaz \& Aguirreolea (2008) estiman que a lo largo de la vida de una planta, se pierde $95 \%$ del agua absorbida por transpiración.

La cantidad de agua que utiliza y transpira la planta depende de la especie y de las condiciones ambientales cambiantes (Gariglio et al. 2007). Evidentemente, los factores ambientales no solamente influyen en los procesos físicos de difusión y de evaporación, sino también en la apertura y en el cierre de los estomas de la superficie foliar, a través de los que pasa el agua y el $\mathrm{CO}_{2}$ (Salisbury \& Ross, 2000). Los factores ambientales más importantes que afectan la transpiración son: radiación solar, déficit de presión de vapor del aire, temperatura, velocidad del viento, concentración de $\mathrm{CO}_{2}$ y disponibilidad de humedad y nutrientes del suelo (Sánchez-Díaz \& Aguirreolea, 2008; Pritchard \& Amthor, 2005).

El objetivo de la presente investigación fue evaluar la tasa de transpiración, la densidad estomática y los factores ambientales de humedad relativa, temperatura e irradiancia, dentro de la copa de árboles de feijoa, con el fin de diferenciar el comportamiento entre hojas del estrato superior e inferior.

\section{MATERIALES Y MÉTODOS}

Localización del área de estudio: El área de estudio está localizada en el municipio de Tibaná (departamento de Boyacá), en la finca El Triunfo, vereda Sírama, ubicada a una altitud de $2.100 \mathrm{msnm}$ y a $5^{\circ} 19^{\prime} 14^{\prime \prime} \mathrm{N}$ y $73^{\circ} 24^{\prime} 02^{\prime \prime} \mathrm{W}$. La temperatura media del sitio fue de $16^{\circ} \mathrm{C}$ y corresponde al Bosque seco, casi húmedo, según la clasificación de zonas de vida de Holdridge (IGAC, 2003), con una precipitación promedio anual de $924 \mathrm{~mm}$ y con régimen pluvial bimodal (marzo-mayo y septiembre-noviembre) (IDEAM, 2007).

Material vegetal: El trabajo de campo, se llevó a cabo en enero de 2010, con árboles de feijoa (variedad no definida debido a la propagación por semilla), de 10 años de edad, sembrados a una distancia de $3 \mathrm{~m}$, entre hileras y $5 \mathrm{~m}$, entre plantas, para una densidad de 650 árboles/ha. Los árboles tuvieron una altura de $2 \mathrm{~m}$ en promedio y la fertilización con macro y micronutrientes, se realizó según la experiencia de los cultivadores de la zona, dos veces por año. En el momento de las mediciones, el suelo se encontró en capacidad de campo (-0,02 MPa).

Densidad estomática: A través de la técnica de esmalte, se realizaron improntas de la parte media del envés de las hojas muestreadas, a las 8 am, previa eliminación de tricomas (Pérez et al. 2010; Sánchez et al. 2013), para observación y conteo de estomas con Microscopio marca Olimpus ${ }^{\circledR}$, objetivo 40X. Posteriormente, se determinó el número de estomas en 5X; este procedimiento se realizó tres veces por campo y se promedió.

Tasa de transpiración y determinación de variables: Los datos, se tomaron aleatoriamente con un porómetro marca Li-Cor ${ }^{\circledR}$ serie Li-1600. Se registró humedad relativa (\% HR), temperatura $\left({ }^{\circ} \mathrm{C}\right)$, irradiancia $\left(\mu \mathrm{mol} \mathrm{m} \mathrm{m}^{-2} \mathrm{~s}^{-1}\right)$ y transpiración $\left(\mu \mathrm{g} \mathrm{cm}^{-2} \mathrm{~s}^{-1}\right)$, de las diferentes hojas. Se muestrearon nueve hojas por estrato (superior e inferior) de la copa. Los valores, se registraron en 7 horas del día (8:00, 9:00, 10:00 y 11:00 am, 12:00 m y 13:00 y 14:00 pm), durante 3 días diferentes, 
presentando los resultados del primer día de las mediciones, con condiciones climáticas típicas de la zona. Las hojas muestreadas fueron identificadas y llevadas al laboratorio para su posterior análisis.

Análisis estadístico: El ensayo, se realizó en forma de un estudio descriptivo. Para la variable número de estomas, se utilizó el programa analítico SAS $®$ versión 9.0, aplicando la prueba t de Student a una probabilidad de $\mathrm{P} \leq 0,001$. Para las demás variables climáticas, se efectuaron correlaciones.

\section{RESULTADOS Y DISCUSIÓN}

Número de estomas: El promedio de estomas presentes en el envés de las hojas del estrato superior de la copa fue de 91 estomas $/ \mathrm{mm}^{2}$ y significativamente mayor $(P \leq 0,001)$, en un $14,3 \%$, comparado con el estrato inferior, con 78 estomas, aunque menor al promedio reportado para otra especie mirtácea, el arazá (Barrera et al. 2009). Friedrich \& Fischer (2000) indican que en el estrato superior, se presenta mínimo sombrío mutuo y las hojas están en pleno crecimiento, lo que favorece el desarrollo de los estomas. Adicionalmente, en el estrato inferior, se presenta menor irradiancia compa- rado con el estrato superior (Tabla 1), lo cual, está relacionado con la densidad estomática observada; en acuerdo a lo reportado por Öpik \& Rolfe (2005), quienes indican que en muchas especies la frecuencia estomática decrece en la medida que disminuye la irradiancia. Martínez-Vega et al. (2008) registraron en árboles de feijoa, 35\% de la radiación solar incidente en la base inferior de la copa, comparado con $90 \%$ de radiación, en la parte superior de la copa. De la misma manera, en arazá, se reporta la presencia de estomas restringidos a la parte abaxial (envés) de la hoja y mayor densidad en hojas desarrolladas a alta irradiancia, en comparación con baja irradiancia (Barrera et al. 2009).

Transpiración: Los rangos promedio de transpiración fueron variables: en el estrato superior, entre 1,2 y $2,2 \mathrm{~g} \mathrm{H}_{2} \mathrm{O} \mathrm{cm}^{-2} \mathrm{~s}^{-1}$, mientras que en el dosel inferior, entre 0,5 y $1,7 \mathrm{~g} \mathrm{H}_{2} \mathrm{O} \mathrm{cm}$ $\mathrm{s}^{-1}$ (Tabla 1); encontrándose dentro del rango reportado para arazá (Barrera et al. 2009) y otra mirtácea, la guayaba (Solarte et al. 2010). Las tasas de transpiración promedio, más altas en el estrato superior, concuerdan con Kramer (1989), quien atribuye el aumento de la transpiración a la mayor cantidad de estomas presentes en las hojas más expuestas al sol. Por otra parte, se ha reportado que, a mayor irradiancia

Tabla 1. Condiciones de humedad relativa, temperatura, irradiancia y transpiración en un huerto de feijoa en Tibaná, Boyacá; \pm desviación estándar.

\begin{tabular}{|c|c|c|c|c|c|}
\hline Estrato & Hora & $\begin{array}{c}\text { Humedad } \\
\text { rel. (\%) }\end{array}$ & $\begin{array}{c}\text { Temperatura } \\
\left({ }^{\circ} \mathrm{C}\right)\end{array}$ & $\begin{array}{c}\text { Irradiancia } \\
\left(\mu \mathrm{mol} \mathrm{m} \mathrm{m}^{-2} \mathrm{~s}^{-1}\right)\end{array}$ & $\begin{array}{l}\text { Transpiración } \\
\left(\mu \mathrm{g} \mathrm{H}_{2} \mathrm{O} \mathrm{cm}^{-2}\right. \\
\left.\mathrm{s}^{-1}\right)\end{array}$ \\
\hline \multirow{8}{*}{ Superior } & 08:00 & $68,7 \pm 1,5$ & $14,7 \pm 0,9$ & $88,3 \pm 21,1$ & $1,713 \pm 0,700$ \\
\hline & 09:00 & $60,3 \pm 0,4$ & $16,8 \pm 0,2$ & $331,7 \pm 87,7$ & $1,192 \pm 0,053$ \\
\hline & $10: 00$ & $53,9 \pm 0,2$ & $18,2 \pm 0,1$ & $306,7 \pm 31,1$ & $1,981 \pm 1,164$ \\
\hline & $11: 00$ & $46,3 \pm 1,9$ & $22,8 \pm 1,4$ & $623,3 \pm 95,5$ & $2,980 \pm 0,913$ \\
\hline & $12: 00$ & $38,0 \pm 0,5$ & $28,1 \pm 1,6$ & $1526,7 \pm 124,4$ & $2,210 \pm 1,297$ \\
\hline & $13: 00$ & $32,8 \pm 0,3$ & $27,6 \pm 1,8$ & $1076,7 \pm 444,4$ & $2,239 \pm 0,460$ \\
\hline & $14: 00$ & $34,8 \pm 0,3$ & $24,7 \pm 0,8$ & $543,0 \pm 142,0$ & $2,025 \pm 0,943$ \\
\hline & Promedio & $47,8 \pm 0,7$ & $21,8 \pm 1,0$ & $642,3 \pm 135,2$ & $2,048 \pm 0,790$ \\
\hline \multirow{8}{*}{ Inferior } & 08:00 & $66,7 \pm 1,5$ & $14,6 \pm 0,8$ & $19,7 \pm 8,2$ & $0,486 \pm 0,063$ \\
\hline & 09:00 & $59,3 \pm 0,9$ & $16,1 \pm 1,1$ & $63,3 \pm 15,5$ & $0,762 \pm 0,015$ \\
\hline & $10: 00$ & $53,9 \pm 0,2$ & $17,6 \pm 0,2$ & $42,3 \pm 25,8$ & $1,000 \pm 0,289$ \\
\hline & $11: 00$ & $46,0 \pm 1,6$ & $21,3 \pm 0,3$ & $128,7 \pm 100,9$ & $1,663 \pm 0,511$ \\
\hline & $12: 00$ & $37,7 \pm 0,9$ & $24,9 \pm 0,3$ & $143,3 \pm 78,9$ & $1,543 \pm 0,358$ \\
\hline & $13: 00$ & $32,8 \pm 0,0$ & $24,3 \pm 0,5$ & $176,0 \pm 196,0$ & $1,350 \pm 0,475$ \\
\hline & $14: 00$ & $34,0 \pm 0,0$ & $23,2 \pm 0,8$ & $117,7 \pm 121,5$ & $1,585 \pm 0,374$ \\
\hline & Promedio & $47,2 \pm 0,9$ & $20,3 \pm 0,6$ & $98,7 \pm 78,1$ & $1,198 \pm 0,298$ \\
\hline
\end{tabular}


en estratos superiores en una planta, mayor la tasa de transpiración (Kozlowski \& Pallardy, 1997).

En la gráfica 1, se observa que la tasa de transpiración es variable, dependiendo del estrato en los árboles, siendo mayor en el estrato superior que en el inferior, similar a lo reportado por Kramer (1989). En general, se encontró que el rango más alto de transpiración, se presenta entre las 10:00 a.m. y 12:00 m. Resultados similares encontraron Galvis et al. (1999) en feijoa cultivada en Suba (Cundinamarca). Igualmente, es hacia el mediodía cuando se registra mayor temperatura e irradiancia y menor humedad relativa, para cada uno de los estratos, respectivamente (Tabla 1), lo cual, provoca después del pico de máxima transpiración, cierre estomático y, por tanto, un descenso en la transpiración y síntesis de fotoasimilados (Kramer, 1989; Galvis et al. 1999).

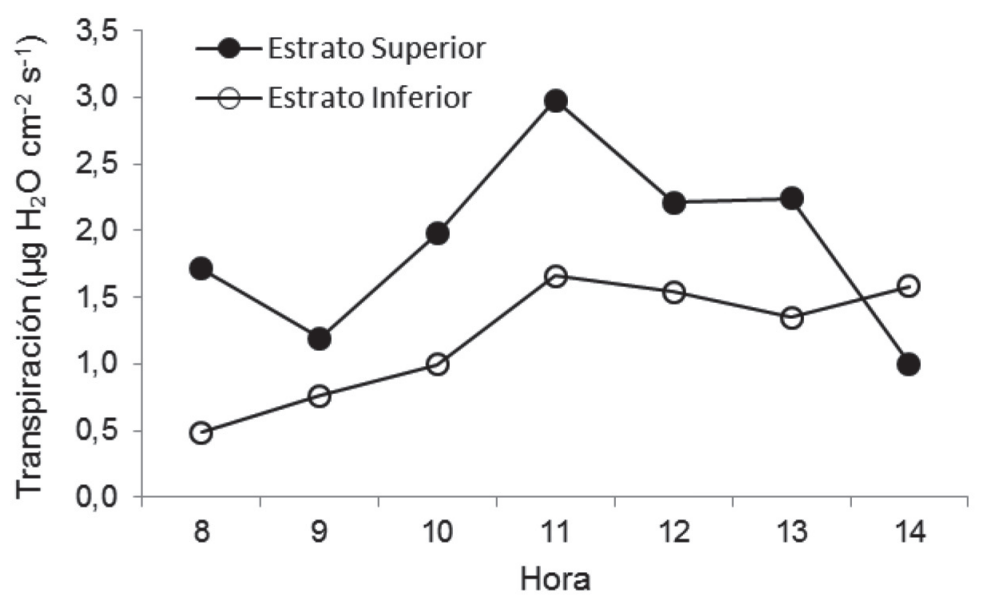

Gráfica 1. Comportamiento de la transpiración en 7 horas diferentes del día, en los dos estratos de la copa de feijoa.

En la interacción de las variables horas y estratos no se observó significancia $(P>0,05)$ lo que indica que el efecto es muy variado, dependiendo de las condiciones ambientales, durante el transcurso del día.

Solarte et al. (2010) observaron que el transcurso de la transpiración depende del microclima del sitio. En variedades de guayaba, en el departamento de Santander (Colombia), localidad Barbosa, se reporta que la mayor tasa de transpiración ocurre a las 10:00 a.m.; en Puente Nacional, ocurre a las 8:00 a.m. y, en Vélez, según la variedad, entre las 9:00 a.m. a $12: 00 \mathrm{~m}$.

En la gráfica 2, se observa para los estratos superior e inferior, relación inversa (correlaciones negativas $R^{2}=-0,3461$ y $R^{2}=-0,8603$, respectivamente), entre humedad relativa y transpiración, es decir, que a medida que disminuye la HR aumenta la transpiración (Tabla 1; Gráfica 2); tal como lo reportado, para otras especies, (Kearns \& Assmann, 1993; Solarte et al. 2010; Barrera et al. 2009). Esta correlación fue mucho más acentuada en el estrato inferior, posiblemente, al microclima en esta parte de la copa, ya que en el dosel superior existe mucha más aireación (Galvis et al. 1999).
Los estomas de muchas especies (aunque no de todas) son muy sensibles a la humedad atmosférica (Tibbitts, 1979) y se cierran cuando la diferencia entre el contenido de vapor del aire y el de los espacios intercelulares supera un nivel crítico. Un gradiente elevado tiende a inducir oscilaciones en la apertura y en el cierre, con una periodicidad de $30 \mathrm{~min}$, aproximadamente, por lo que suponen, Salisbury \& Ross (2000), que cuando un gradiente pronunciado de vapor induce el cierre se agota el $\mathrm{CO}_{2}$ en la hoja, provocando la apertura del estoma. Tanto en el estrato superior como en el inferior, los niveles más altos de transpiración se producen en el rango de 30 a $45 \%$ de HR (Gráfica 2), significando una apertura estomática mayor.

En la gráfica 3, se observa que la temperatura mostró una correlación alta positiva con la transpiración, la cual, varió respecto a los estratos y aumentó a medida que incrementó la temperatura (Gráfica 3). Para el estrato superior, se presentaron promedios de transpiración más altos que en el estrato inferior. Este comportamiento concuerda con Kramer (1989), quien sugiere que un aumento de temperatura conlleva a una mayor transpiración, causada por el incremento del gradiente de presión de vapor entre la hoja y el aire. Cam- 


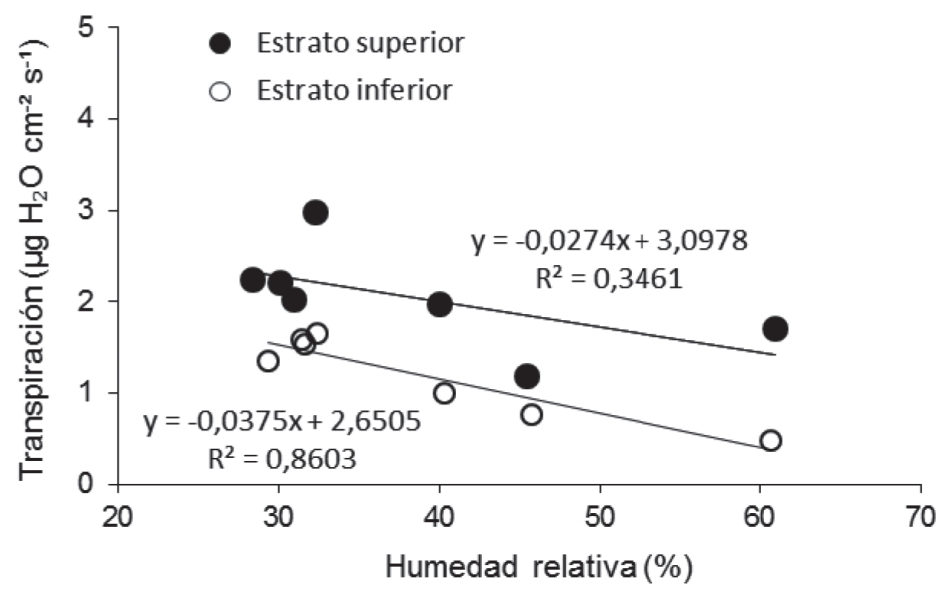

Gráfica 2. Comportamiento de la transpiración en relación con la humedad relativa en los dos estratos de la copa de feijoa.

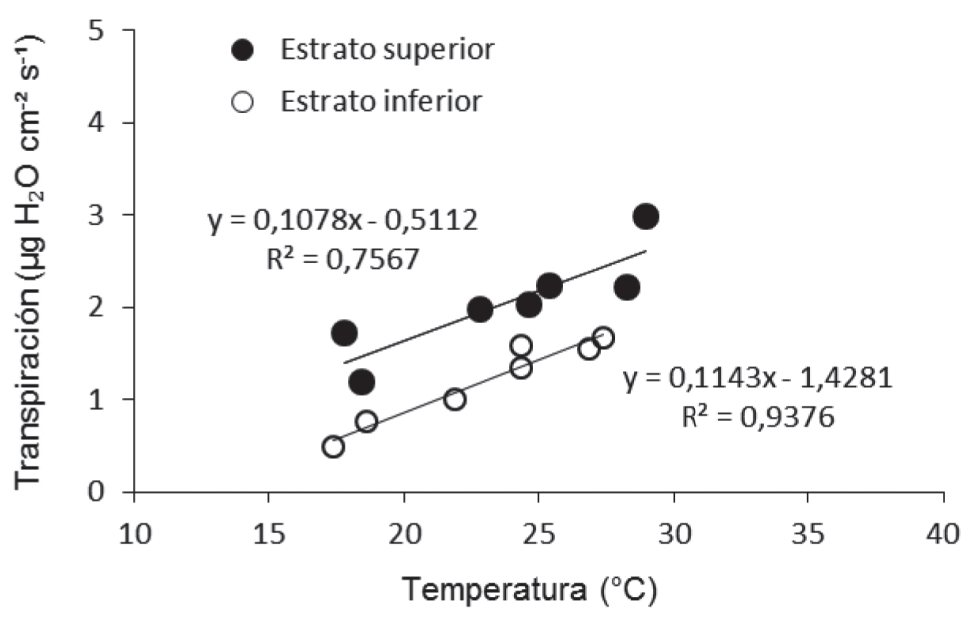

Gráfica 3. Comportamiento de la transpiración en relación con la temperatura en los dos estratos de la copa de feijoa.

bios de la temperatura foliar, inclusive pequeños, son probablemente los más responsables de la mayoría de los cambios de transpiración a corto plazo (Kramer, 1989).

Hall (2001) describió que sobre un cierto rango de temperatura (dependiendo de la especie) el incremento de la temperatura causa una mayor apertura y conductancia estomática (que coincide con el aumento de la transpiración). Cuando existen temperaturas foliares crecientes, como se registraron en el estrato superior de la copa, la permeabilidad de la cutícula aumenta, ocasionando, probablemente, mayor pérdida de vapor de agua por parte de la hoja (Kramer, 1989; Sánchez-Díaz \& Aguirreolea, 2008).
Integrado con los factores ambientales HR y temperatura (Gráficas 2 y 3), la transpiración aumentó con el incremento de la irradiancia (Gráfica 4). En el estrato superior de la copa altas tasas transpiratorias coincidieron con altas irradiancias y similar tendencia se observó en el estrato bajo, pero con menores valores, tanto de irradiancia como de transpiración (Gráfica 4, Tabla 1). El promedio de la irradiancia entre las 8:00 a.m. y 2:00 p.m. fue de $642,3 \mu \mathrm{mol} \mathrm{m}^{-2} \mathrm{~s}^{-1}$, para el estrato superior y de $98,7 \mu \mathrm{mol} \mathrm{m} \mathrm{m}^{-2} \mathrm{~s}^{-1}$, para el inferior (Tabla 1), la cual, es una diferencia mayor a la que encontraron Martínez-Vega et al. (2008) entre los dos estratos, en árboles de feijoa de 6 años de edad. 


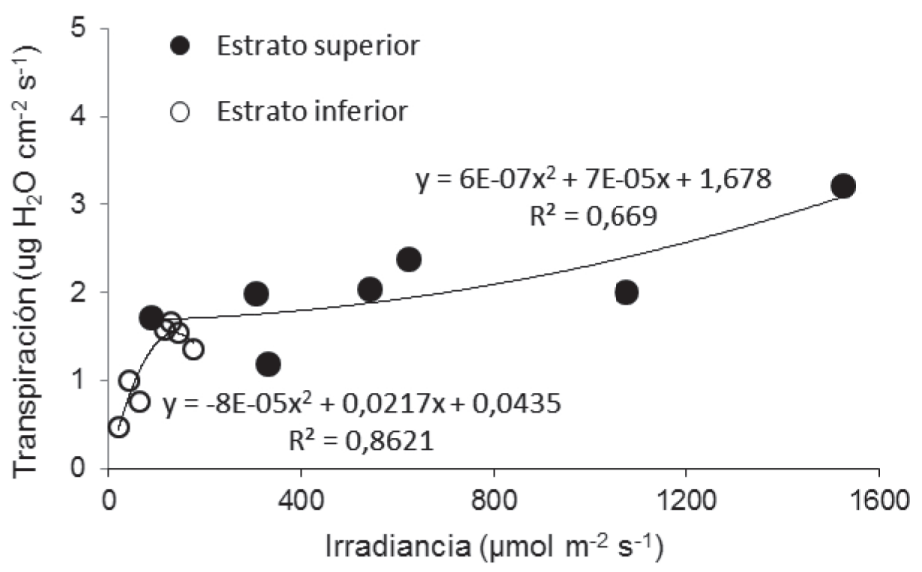

Gráfica 4. Comportamiento de la transpiración en relación con la irradiancia en los dos estratos de la copa de feijoa.

De acuerdo a todo lo anterior, se puede concluir, que la tasa de transpiración frente a los factores ambientales evaluados es mayor en el estrato superior y menor en el estrato inferior, donde los niveles de radiación que inciden en la feijoa son menores, lo que sugiere que, para mejorar el desempeño fisiológico de la planta, se realicen podas que permitan la entrada de luz, eliminando ramas y área foliar sobrantes.

Conflicto de intereses: El manuscrito fue preparado y revisado con la participación de todos los autores, quienes declaramos que no existe ningún conflicto de intereses que ponga en riesgo la validez de los resultados presentados.

\section{BIBLIOGRAFÍA}

1. AGRONET. 2014. Área cosechada’, producción y rendimiento de feijoal, |1992-2012.Disponible desde Internet en: www.Agronet.gov.co (con acceso 05/02/2014).

2. BARRERA, J.; ORJUELA, N.; MELGAREJO, L.M.; CAICEDO, D.; HERNÁNDEZ, M.S. 2009. Efecto de deficiencias minerales y de la luz en arazá (Eugenia stipitata) y copoazú (Theobroma grandiflorum). En: Hernández, M.S., Barrera, J. (Comp.). Frutas amazónicas: competitividad e innovación. Editorial Instituto Amazónico de Investigaciones Científicas Sinchi, Bogotá. p.11-34.

3. BARRERO, F. 1993. La ecotecnología en el contexto de la extensión y el desarrollo rural. AgroDesarrollo 4(12):104-117.

4. FISCHER, G. 2003. Ecofisiología, crecimiento y desarrollo de la feijoa. En: Fischer, G.; Miranda, D.; Cayón,
G.; Mazorra, M. (eds.). Cultivo, poscosecha y exportación de la feijoa (Acca sellowiana Berg). Produmedios (Bogotá). p.9-26.

5. FRIEDRICH, G.; FISCHER, M. 2000. Physiologische Grundlagen des Obsbaues. Verlag Eugen Ulmer (Stuttgart, Alemania). 512p.

6. GALVIS, J., HERNÁNDEZ, M.S.; FISCHER, G. 1999. Transpiración de la feijoa (Acca sellowiana Burret) en la Sabana de Bogotá. Rev. Comalfi 26(1-3):5661.

7. GARIGLIO, N.F.; PILATTI, R.A.; FONFRÍA, M.A. 2007. Requerimientos ecofisiológicos de los árboles frutales. En: Sozzi, G.O. (ed.). Árboles frutales. Ecofisiología, cultivo y aprovechamiento. Editorial Facultad de Agronomía, Universidad de Buenos Aires (Argentina). p.41-82.

8. HALL, A.E. 2001. Crop responses to environment. CRC Press (Boca Raton, FL). 228p.

9. IDEAM. 2007. Instituto de hidrología, meteorología y estudios ambientales. Sistema de información registros estacionales Corpochivor (Tibaná, Boyacá, Colombia).

10. IGAC. 2003. Mapa suelos de Colombia, archivo de computador escala 1:500000: Memoria explicativa. Instituto Geográfico Agustín Codazzi (Bogotá).

11. KEARNS, E.V.; ASSMANN, S.M. 1993. The guard cellenvironment connection. Plant Physiol. 102:711715. 
12. KOZLOWSKI, T.T.; PALLARDY, S.G. 1997. Physiology of woody plants. 2nd ed. Academic Press (San Diego, CA). 411p.

13. KRAMER, P. 1989. Relaciones hídricas de suelos y plantas. Editorial Harla (México). 538p.

14. LÓPEZ, Y. 2000. Relaciones hídricas en el continuo agua-suelo-planta-atmosfera. Editorial Universidad Nacional de Colombia (Palmira). 88p.

15. MARTÍNEZ-VEGA, R.R.; FISCHER, G.; HERRERA, A.; CHAVES, B.; QUINTERO, O.C. 2008. Características físico-químicas de frutos de feijoa influenciadas por la posición en el canopi. Rev. Colomb. Cienc. Hortic. 2(1):21-32.

16. ÖPIK, H.; ROLFE, S. 2005. The physiology of flowering plants. Cambridge University Press (Cambridge, UK). 392p.

17. PÉREZ, L.V.; ROJAS, Y.A.; MELGAREJO, L.M. 2010. Agua. En: Melgarejo, L.M. (Ed.) Experimentos en fisiología vegetal. Departamento de Biología, Facultad de Ciencias, Universidad Nacional de Colombia, Bogotá. p.63-77.

18. PRITCHARD, S.G.; AMTHOR, J.S. 2005. Crop and environmental change. Food Product Press (New York, NY). 421p.

19. QUINTERO, O.C. 2012. Feijoa (Acca sellowiana Berg). En: Fischer, G. (ed.). Manual para el cultivo de frutales en el trópico. Produmedios (Bogotá). p.443473.
20. SALISBURY, F.; ROSS, C. 2000. Fisiología de las plantas. Vol. 1. Paraninfo-Thomson Learning (Madrid). 305p.

21. SÁNCHEZ, C.; FISCHER, G.; SANJUANELO, D.W. 2013. Stomatal behavior in fruits and leaves of the purple passion fruit (Passiflora edulis Sims) and fruits and cladodes of the yellow pitaya [Hylocereus megalanthus (K. Schum. ex Vaupel) Ralf Bauer]. Agron. Colomb. 31(1):38-47.

22. SÁNCHEZ-DÍAZ, M.; AGUIRREOLEA, J. 2008. Transpiración y control estomático. En: Azcón-Bieto, J.; Talón, M. (Eds.). Fundamentos de fisiología vegetal. McGraw-Hill Interamericana de España (Madrid). p.41-56.

23. SCHUMANN, M.; LÜDDERS, P. 1992. Cultivation of feijoa: Possibilities and limits in Colombia. Erwerbsobstbau 34(4):110-112.

24. SOLARTE, M.E.; ROMERO, H.M.; MELGAREJO, L.M. 2010. Caracterización ecofisiológica de la guayaba de la hoya del río Suárez. En: Morales, A.L.; Melgarejo, L.M. (Eds.). Desarrollo de productos funcionales promisorios a partir de la guayaba Psidium guajava L.) para el fortalecimiento de la cadena productiva. Facultad de Ciencias, Universidad Nacional de Colombia (Bogotá). p.25-56.

25. TIBBITTS, T.W. 1979. Humidity and plants. Bioscience. 29:358-363.

Recibido: Septiembre 28 de 2013

Aceptado: Abril 1 de 2014

Como citar

Naizaque, J.; García, G.; Fischer, G.; Melgarejo, L.M. 2014. Relación entre la densidad estomática, la transpiración y las condiciones ambientales en feijoa (Acca sellowiana [O. Berg] Burret). Rev. U.D.C.A Act. \& Div. Cient. 17(1): 115-121. 
